\title{
Osteotomies for Hallux Valgus
}

\author{
Bill GX Zhang ${ }^{1}$, Terence Chin $^{2}$
}

\begin{abstract}
Hallux valgus (HV) is a common forefoot condition and patients frequently present for surgical correction. Despite many years of collective surgical experience, a variety of first metatarsal osteotomies and techniques are available to the orthopedic surgeon with no consensus of a preferred technique. How does one navigate the field of differing osteotomies for HV? Are there some osteotomies with certain advantages over others? This paper seeks to answer these questions and will discuss the pathoanatomy of HV, the principles and objectives of surgical correction, and the osteotomies currently in popular use in orthopedic practice.

Keywords: Bunion, Forefoot, Hallux valgus, Orthopedics, Osteotomy, Reconstruction, Surgery, Surgical correction.

Journal of Foot and Ankle Surgery (Asia Pacific) (2020): 10.5005/jp-journals-10040-1128
\end{abstract}

\section{INTRODUCTION}

Hallux valgus (HV) is a common condition characterized by lateral angulation and often pronation of the hallux. It is a significant and often underappreciated source of pain and dysfunction. Surgical correction of HV includes realignment osteotomies of the first metatarsus (MT) and first proximal phalanx (PP) with associated soft tissue correction. Despite over 100 years of surgical correction of HV, and more than 100 different types of techniques and osteotomies described, surgical treatment for this common condition continues to evolve. ${ }^{1,2}$

In addition to "open" surgery, minimally invasive surgery (MIS) for HV correction, not a new concept, having been popular in parts of Europe in the 1970s, is also becoming more popular and de rigueur for some foot and ankle surgeons today. ${ }^{3}$ Minimally invasive surgery $\mathrm{HV}$ correction will only be briefly mentioned here and readers are referred to the MIS paper in the current edition of this journal for further details.

Clearly, there are several first MT osteotomies that can be effective for HV correction. No single osteotomy is perfect. A surgeon's preferred osteotomy is determined largely by his/her training, past, and continuing experience. Another factor for the different techniques and osteotomies reported is that HV constitutes a non-homogenous group of deformities with varying degrees of severity, presence of abnormal distal metatarsal articular angles (DMAA), and whether there is associated arthritis and stiffness. Not all HV are equal, and one needs to assess each HV on its merits and select the osteotomy/technique (or even consider fusion procedures) which is best placed to achieve the desired correction.

This paper outlines the principles of HV correction and discusses the strengths and weaknesses of different types of osteotomies, with an emphasis on the scarf osteotomy

\section{Pathoanatomy and Principles of Correction}

The surgical treatment of HV begins first with a thorough understanding of the pathoanatomy. In its simplest terms, $\mathrm{HV}$ is lateral angulation of the hallux. Although the exact sequence of events in the causative chain is still unclear, the salient features of HV include medial deviation of the first MT and lateral angulation of the first PP. ${ }^{1}$ These manifest radiologically as an increased intermetatarsal angle (IMA) and hallux valgus angle (HVA) on weight-bearing foot X-rays ${ }^{4}$
${ }^{1}$ Department of Orthopaedics, Austin Health, Heidelberg, Victoria, Australia

${ }^{2}$ Department of Orthopaedics, Austin Health, Heidelberg, Victoria, Australia; St Vincent's Private, Melbourne, Victoria, Australia

Corresponding Author: Terence Chin, Department of Orthopaedics, Austin Health, Heidelberg, Victoria, Australia; St Vincent's Private, Melbourne, Victoria, Australia, Phone: +613 91165827, e-mail: terencechin03@gmail.com

How to cite this article: Zhang BGX, Chin T. Osteotomies for Hallux Valgus. J Foot Ankle Surg (Asia Pacific) 2020;7(2):38-45.

Source of support: Nil

Conflict of interest: $\mathrm{Mr}$ Terence Chin is a paid consultant for Ozextremities. He also receives royalties from CAB Medical.

(Fig. 1A). The first PP often subluxates laterally over the first MT head. This is often accompanied by a pronation deformity of the first ray. ${ }^{5,6}$ The medial capsule becomes attenuated and the lateral sesamoidmetatarsal (MT)-PP ligament complex contracts (Fig. 1B). ${ }^{2,5}$ As the HV progresses, the line of pull of extensor hallux longus (EHL) and flexor hallux longus ( $\mathrm{FHL}$ ) displaces lateral to the long axis of the first ray to become further deforming forces, worsening the HV (Fig. 1C). ${ }^{5,7}$ With further medial drift of the first MT, the MT head subluxates over the sesamoids, eroding the crista on the plantar aspect of the MT head, and leaving the sesamoids (which remain static in their original position) to lie within the first web space (Fig. 1C). ${ }^{8}$

In younger patients with juvenile $\mathrm{HV}$, the deformity is often different from adults. There is frequently a lateral deviation of the articular surface of the first MT head without loss of joint congruity. This presents as an increased in the DMAA, and the IMA may be preserved (Fig. 1D). ${ }^{9}$ Surgical correction therefore must address the laterally deviated articular surface (increased DMAA).

The aim of surgery is to address the pathoanatomical abnormalities explained above. This involves the following steps (Figs $2 \mathrm{~A}$ and $\mathrm{B}$ ):

- Reduction of the increased IMA and HVA, through first MT and PP osteotomies.

- Correction of any increased DMAA by incorporating a derotational component into the osteotomies performed.

- Release of the tight lateral sesamoid-MT-PP ligament complex (Fig. 2C).

(0) The Author(s). 2020 Open Access This article is distributed under the terms of the Creative Commons Attribution 4.0 International License (https://creativecommons. org/licenses/by-nc/4.0/), which permits unrestricted use, distribution, and non-commercial reproduction in any medium, provided you give appropriate credit to the original author(s) and the source, provide a link to the Creative Commons license, and indicate if changes were made. The Creative Commons Public Domain Dedication waiver (http://creativecommons.org/publicdomain/zero/1.0/) applies to the data made available in this article, unless otherwise stated. 

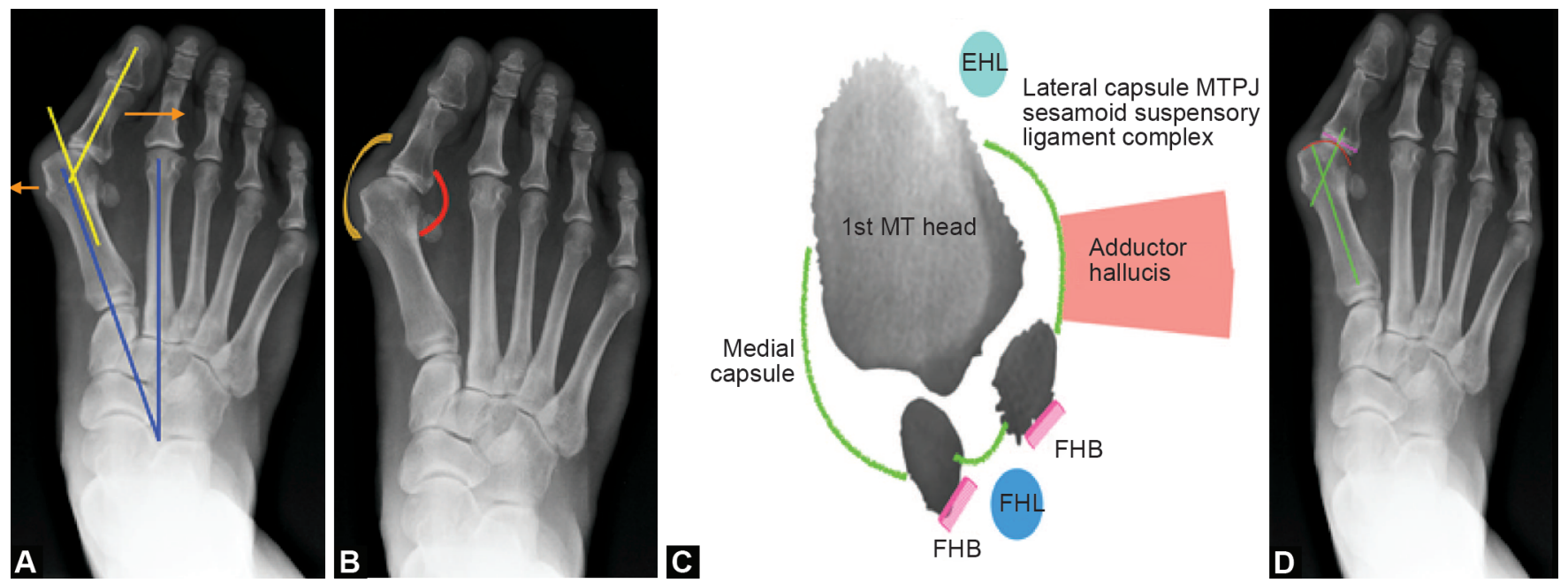

Figs 1 A to D: Radiographs and schematic diagrams illustrating key features of hallux valgus (HV). (A) HV deformity characterized by medial angulation of first metatarsus (MT) and lateral deviation of proximal phalanx (orange arrows). There is an increased intermetatarsal angle (angle subtended by blue lines) and increased hallux valgus angle (the angle subtended between yellow lines); (B) Deficiency of the medial capsule of the first metatarsophalangeal joint (MTPJ) (orange line), with associated contracture of the lateral capsule and lateral sesamoid-MT-proximal phalanx (PP) complex (red line); (C) Schematic diagram illustrating the medial translation of the metatarsal head over the sesamoids leading to erosion of the crista over medial sesamoid, contraction of the lateral sesamoid-MT-PP complex and attenuation of the medial capsule. Flexor hallux brevis (FHB) (lateral head), flexor hallux longus (FHL) and extensor hallux longus (EHL) come to lie lateral to the long axis of the metatarsus and become forces further exacerbating lateral angulation of the proximal phalanx; (D) HV deformity with associated increased distal metatarsal articular angle (angle subtended by green lines). The curved red line demonstrates the articular surface which is laterally angulated to the long axis of the first MT
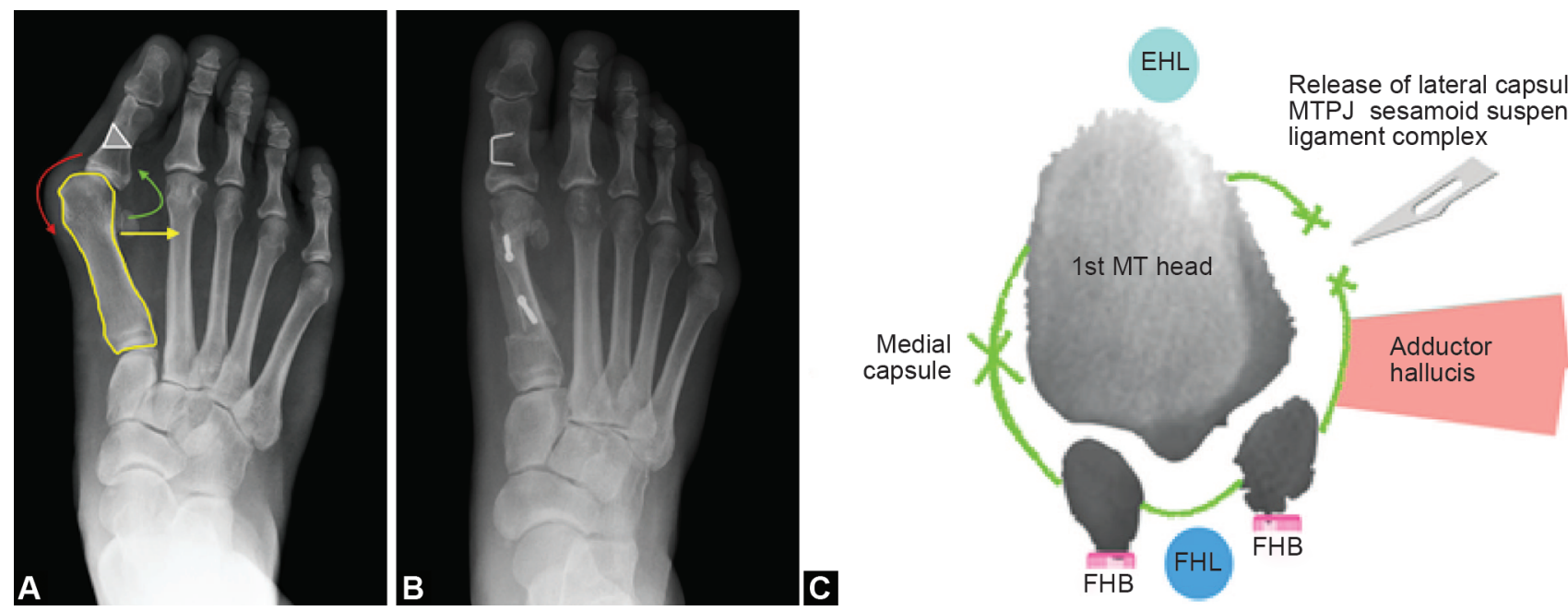

Figs 2A to C: Principles of correction of hallux valgus (HV). (A) Steps in HV correction: Release of lateral sesamoid-metatarsus-proximal phalanx (MT-PP) complex (green arrow); First metatarsal osteotomy with lateral translation of the MT, with / without internal rotation of the capital fragment to correct any distal metatarsal articular angle deformity (yellow arrow); Imbrication of the medial capsule (red arrow); Akin medial closing wedge osteotomy with/without derotation to correct any pronation deformity (white triangle); (B) Radiograph of same patient as Figure 2A following lateral release, scarf and Akin osteotomy and medial plication; (C) Schematic diagram of release of lateral sesamoid-MT-PP ligament complex. This reduces the lateral pulling force on the sesamoid complex and creates an opening for lateral translation of the MT head helping to restore the MT head over the sesamoids

- Plication of the attenuated medial capsule of the first metatarsophalangeal joint (MTPJ).

The final result should ideally be a correction of the IMA and HVA, a restoration of the first MT head over the sesamoids, and a congruent first MTPJ with balanced tensioning of the medial and lateral capsular structures.

\section{Types of First Metatarsus Osteotomies}

Many different first MT osteotomies have been described and can be categorized by the location and the configuration of the osteotomies on the first MT.
These include

- Distal first MT osteotomies-Distal chevron ${ }^{10,11}$ (Fig. 3A), step cut Mitchell, ${ }^{12}$ and oblique Wilson. ${ }^{13}$

- Diaphyseal osteotomies-Mau, ${ }^{14}$ Ludloff $^{15}$ procedures.

- Metadiaphyseal osteotomies-Scarf osteotomy ${ }^{16}$ (Fig. 3B).

- Proximal osteotomies-Proximal chevron, ${ }^{17}$ crescentic, $^{18}$ (Fig. 3C), and medial opening wedge ${ }^{19}$ osteotomies.

It is beyond the scope of this paper to discuss all available osteotomies. The body of experience, however, bears out certain key points. Distal osteotomies are best suited for mild HV as they afford less corrections due to the limited lateral translation of the 


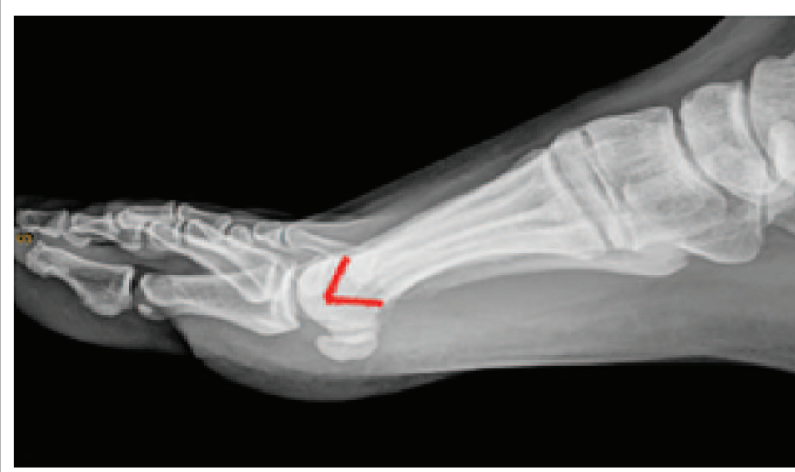

A
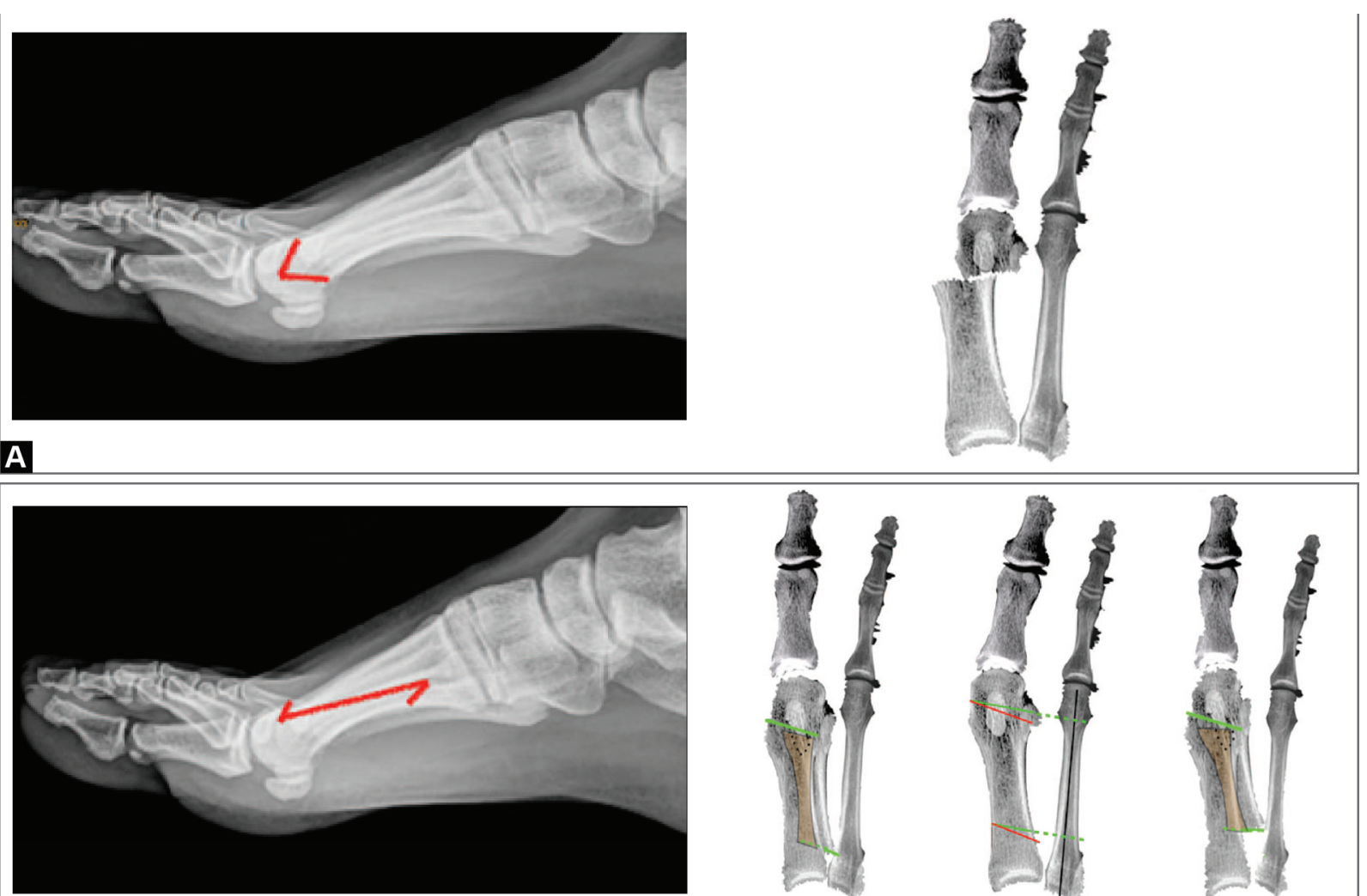

$\mathbf{B}$
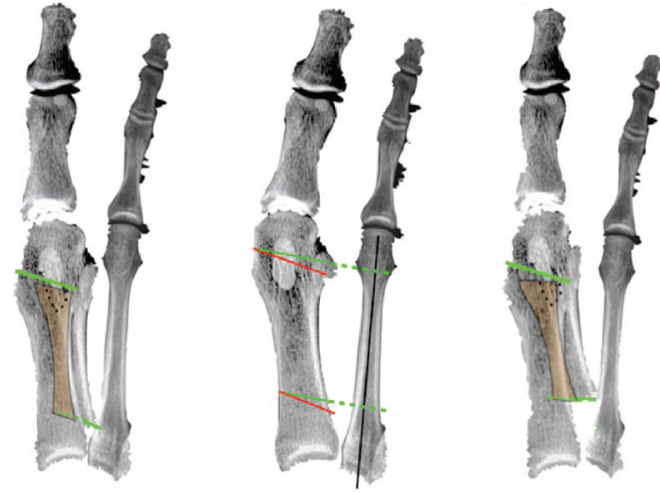

Lateral translation ii

Shortening iii

Rotation iv

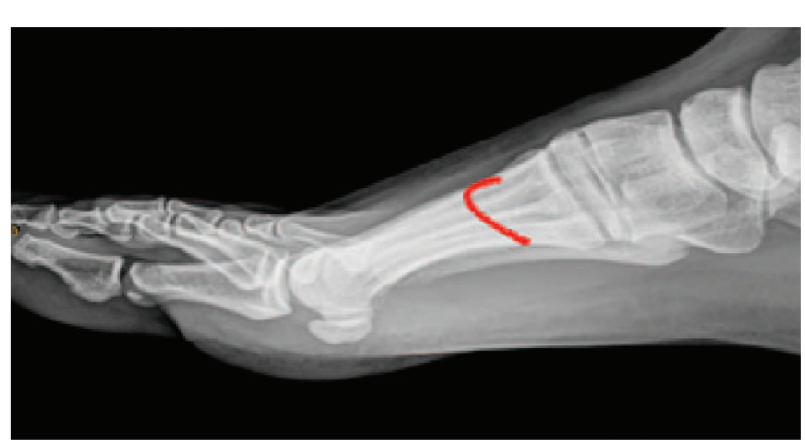

C

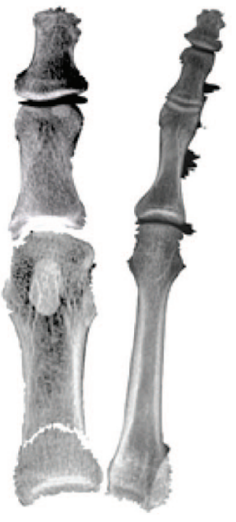

Figs $3 \mathrm{~A}$ to C: Radiographs and schematic diagrams of different types of first metatarsal osteotomies. The red and green lines on radiographs represent the location and configuration of the bone cuts. (A) Distal Chevron osteotomy; (B) Scarf osteotomy. Note the Z-shape osteotomy on the radiograph which begins with a transverse osteotomy dorsally in the distal metaphysis, with the longitudinal section extending from dorsal-distal to proximal-plantar in the metaphysis. Lateral translation of the capital/plantar fragment to correct the intermetatarsal angle and hallux valgus angle. The transverse limbs of the scarf osteotomy are typically angled perpendicular to the longitudinal axis of the second metatarsal (MT) shaft (green lines). Angling the transverse limbs more obliquely and proximally (red lines) will shorten the first MT which is often desirable for severe HV correction. Internal rotation of the capital/plantar fragment can be incorporated to correct distal metatarsal articular angle abnormalities; (C) Crescentic osteotomy

distal first MT fragment. Distal osteotomies also have a higher risk of avascular necrosis (AVN) of the MT head due to the proximity of the nutrient vessels to the osteotomy site. ${ }^{20}$ This is especially the case when the osteotomy is accompanied with excessive soft tissue stripping or lateral release. ${ }^{21}$ Diaphyseal and proximal osteotomies are more suited to moderate to severe HV as they allow for greater lateral translation of the distal metatarsal fragment. ${ }^{17-19}$ Osteotomies based on the proximal metaphysis (proximal chevron and basal crescentic), however, tend to be more unstable due to the longer lever arm of the ground reaction vector tending to displace the osteotomy dorsally. This may lead to dorsal malunion of the first MT with resultant transfer metatarsalgia. ${ }^{22}$

If a DMAA deformity is also present, rotation of the first $M T$ head in the transverse plane is required to achieve a congruent first MTPJ (Fig. 4). ${ }^{23}$ An incongruent first MTPJ (despite improvement the hallux alignment) predisposes to HV recurrence, joint stiffness, and potentially early degenerative change. ${ }^{24}$ Various techniques are available to address an 


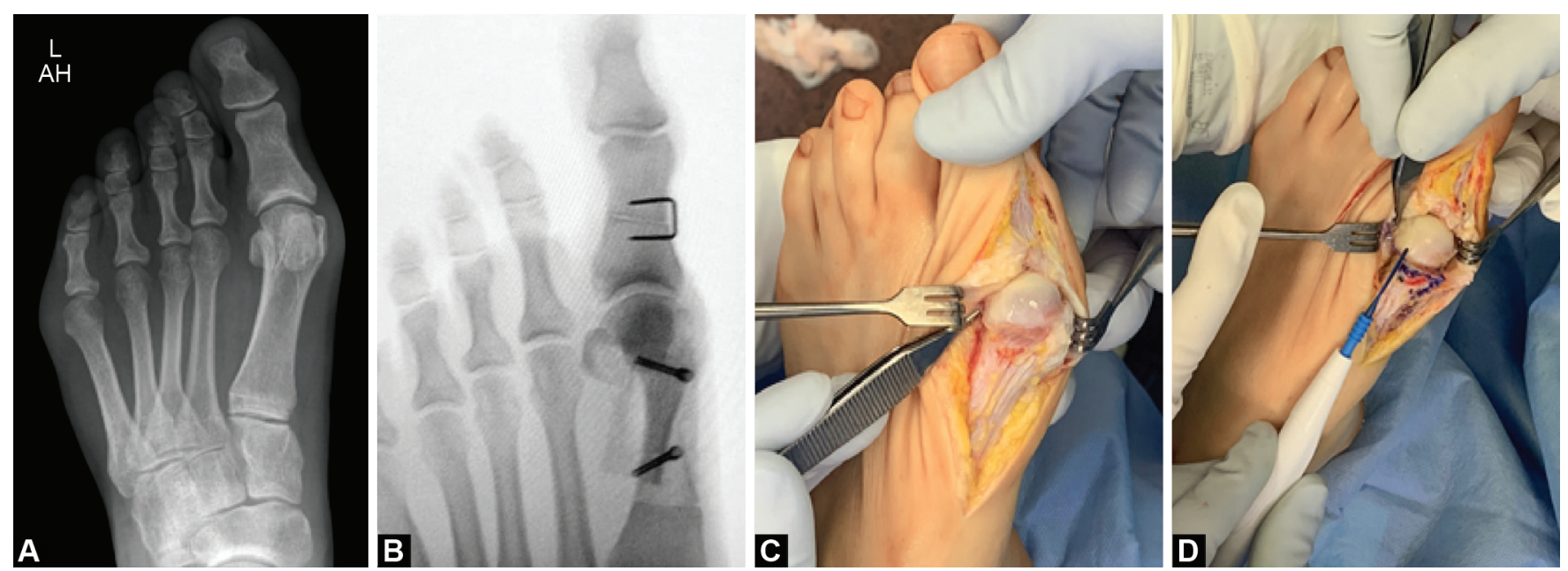

Fig 4A to D: Correction of distal metatarsal articular angle (DMAA) using rotational scarf osteotomy. (A) Preoperative radiograph of a 29-year-old woman with hallux valgus. Note the relatively normal intermetatarsal angle with an increased DMAA; (B) Intraoperative image intensifier picture of rotational scarf osteotomy performed on the same patient. Note the restoration of relative joint alignment to the first metatarsal (MT) axis after surgery; ( $C$ and $D)$ Intraoperative pictures showing the lateral angulation of the articular surface in relation to long axis of the MT shaft (increased DMAA), with subsequent correction
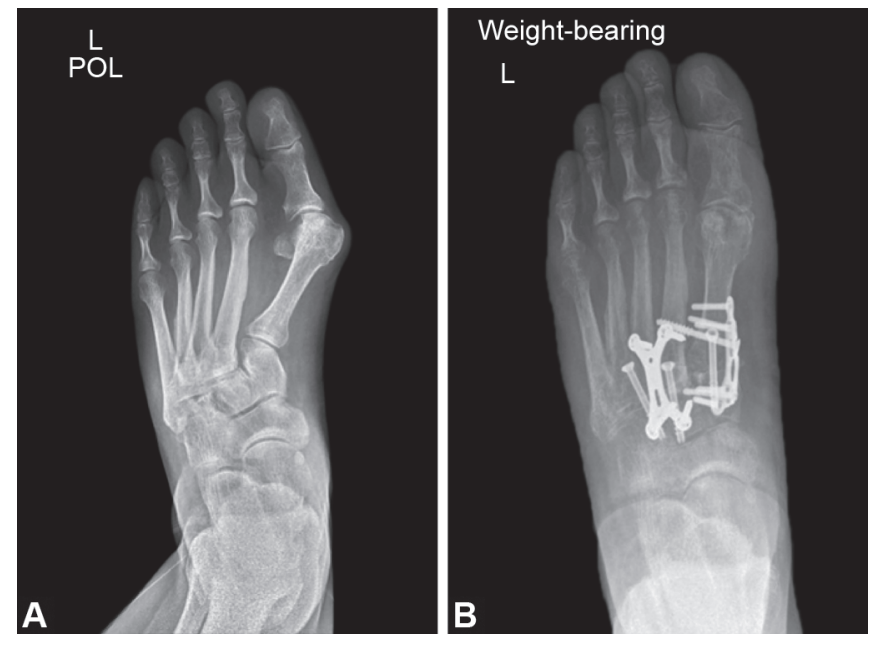

Figs $5 \mathrm{~A}$ and $\mathrm{B}$ : Preoperative and postoperative radiographs of patient with severe hallux valgus with associated metatarsus adductus who underwent Lapidus procedure and second and third TMTJ fusion. (A) Preoperative radiograph; (B) Postoperative radiograph. Note the correction of the IMA, HVA and DMAA. (Courtesy: Mr Andrei Cornoiu)

increased DMAA, such as a biplanar chevron osteotomy and rotational scarf osteotomy (Fig. 4). ${ }^{25,26}$

In patients with severe HV that is deemed not amenable to corrective osteotomies, or HV associated with arthritis of the first MTPJ (hallux rigidus or inflammatory arthropathy), a first MTPJ fusion is the preferred option. ${ }^{27} \mathrm{~A}$ first MTPJ fusion is also appropriate for severe HV in elderly patients with low functional demands. ${ }^{28,29}$

It is clear that a component of $\mathrm{HV}$ results from medial angulation or drift of the first MT. The source of this deformity must therefore, in the absence of angulation through the first MT itself, arise from the first MT-medial cuneiform joint (first TMT joint). The concept of hypermobility of the first TMT joint has been put forward by some as the prime etiological factor in $\mathrm{HV}^{30}$ A corrective fusion of the first TMTJ (Lapidus procedure) has therefore been proposed as a procedure of choice by some surgeons with favorable results. ${ }^{30}$ This concept and the use of a first TMT fusion as a first-line treatment for HV is not universally held, however. Furthermore, a first TMT joint fusion can be difficult to perform, with high nonunion rates reported by some authors. ${ }^{31,32}$ The author's preference is to reserve the first TMT fusion for patients with severe HV, particularly those associated with metatarsus adductus, or in the setting of HV with first TMT joint arthritis (Fig. 5).

\section{Which Osteotomy to Use?}

Ideally, the chosen first MT osteotomy for HV correction should allow for maximal translation of the distal fragment and be capable of correcting a spectrum of deformities ranging from mild to severe HV. It should address the HV deformity on all three planesTransverse (DMAA, IMA, HVA), sagittal, and frontal (pronation/ supination). The resulting construct should be mechanically robust, and allow for stable internal fixation to prevent loss of position and withstand early postoperative heel weight-bearing without the need for rigid splinting/casting.

In the author's experience, the scarf osteotomy fulfils many of these requirements and is the preferred open (non-minimally invasive) osteotomy for HV correction. The word "scarf" is a carpentry term to describe a Z-shape cut used to lengthen beams. It was first described by Lowell Weil and later popularized by Louis Barouk Sr. The osteotomy consists of distal and proximal transverse limbs connected by a longitudinal oblique limb (Fig. 3B). ${ }^{16,33}$ The longitudinal component of the osteotomy begins on the distaldorsal metaphysis and ends at the plantar aspect of the proximal metaphysis, i.e., distal dorsal to proximal plantar [Fig. 3B(i)]. The distal transverse osteotomy is typically directed perpendicular to the second metatarsal neck. The proximal transverse osteotomy parallels its distal counterpart. The capital fragment is then translated laterally to correct the IMA [Fig. 3B(ii)]. Two screws are then used for fixation.

The scarf osteotomy is a versatile osteotomy, permitting correction in multiple planes. These include

- Lateral translation of the MT head fragment by up to 50 to $75 \%$ of width of the shaft, allowing for significant deformity correction and restoration of IMA [Fig. 3B(ii)]. ${ }^{34}$

- Shortening of the first MT, performed either by angling the transverse cuts of the osteotomy more obliquely or by removing 


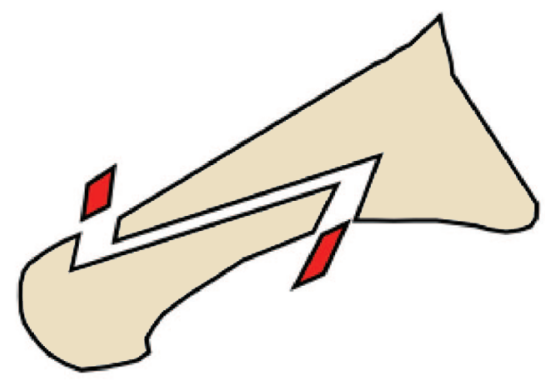

A

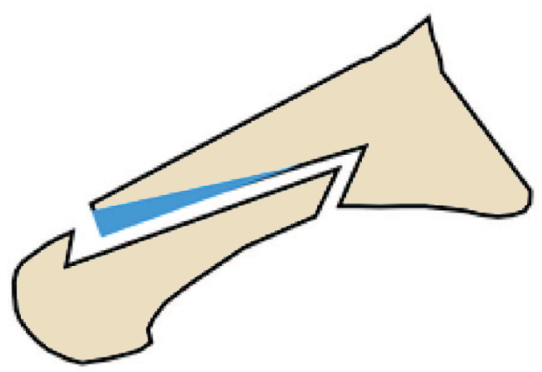

B

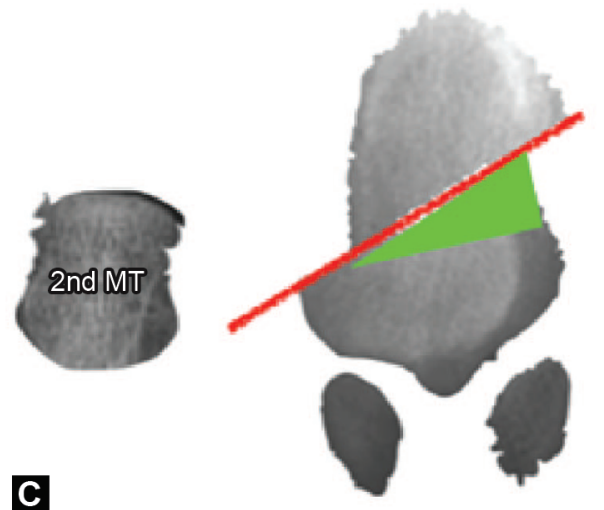

C

Figs 6A to C: Scarf osteotomy is a versatile technique capable of shortening, dorsiflexing and derotating the first metatarsus (MT). (A) Shortening of the first MT by removing segments of bone from the transverse osteotomies (red segments); (B) Elevation of the 1st MT can be achieved by removing a wedge of bone from the dorsal fragment of the scarf osteotomy (blue triangle); (C) Pronation of the hallux can be corrected by removing a wedge of bone from the medial aspect of plantar fragment of the scarf osteotomy (green triangle)
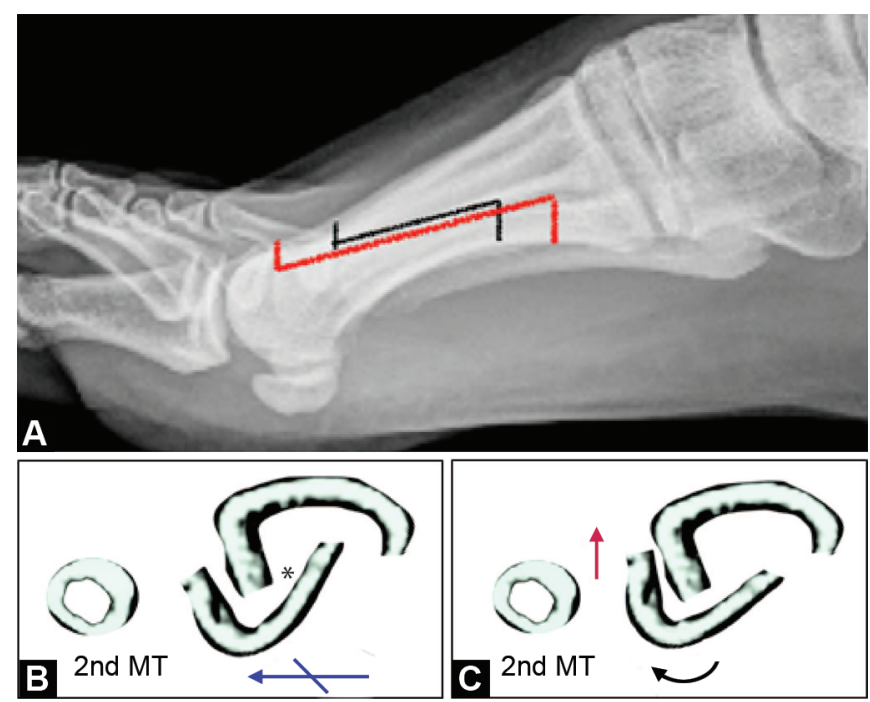

Figs 7A to C: Troughing in scarf osteotomies. (A) A scarf osteotomy which is purely diaphyseal (black line), and does not include the broad distal and proximal metaphysis (cf. red line) results in a higher risk of troughing; ( $\mathrm{B}$ and $\mathrm{C}$ ) When troughing occurs, the dorsal and plantar fragments of the osteotomy become 'locked' (asterisks) and this limits lateral translation (blue arrow) and leads to pronation (black arrow) and elevation of the metatarsal head (red arrow). MT, metatarsus

a segment of bone from the transverse cuts. Shortening of the first ray is often required for moderate to severe $\mathrm{HV}$ corrections [Fig. 3B(iii) and Fig. 6A]. ${ }^{34,35}$

- Correction of any DMMA deformity by internal rotation of the capital fragment, redirecting the articular surface of the first MT so that it is aligned more perpendicular to the long axis of the first MT shaft [Fig. 3B(iv) and Fig. 4]. ${ }^{25,34}$

- Lengthening of the first MT, however, this is rarely required.

- Elevation of the first MT may be required in the cavus foot with associated HV. This is achieved with a distal closing wedge osteotomy with bone removed from the dorsal fragment (Fig. 6B).

- Supination of the osteotomy by removing a wedge of bone from the medial aspect of the plantar fragment (Fig. 6C). Note that any supination posture of the hallux can also be addressed by derotation of the first PP osteotomy (see below).

An important technical point while performing the scarf osteotomy is to ensure the longitudinal cut of the osteotomy be parallel to the oblique plantar surface of the first MT shaft. ${ }^{34}$ To achieve this, the longitudinal cut is commenced on the dorsomedial portion of the shaft and neck, and angled in a plantar direction (Fig. 6C). This is advantageous in that it preserves the majority of the lateral surface of the first MT, allowing for a strong bony strut on which to support the osteotomy, to permit maximal lateral translation (up to $50-75 \%$ of the width) of the first MT shaft. It also acts to lower the first MT head which is beneficial in patients with moderate to severe HV where the first ray has often lost its weight-bearing ability, leading to second ray transfer metatarsalgia. ${ }^{34}$

Potential pitfalls during the procedure include difficulty with fragment translation and troughing. If fragment translation does not occur easily during the surgery, forceful manipulation may cause an iatrogenic fracture to the MT head. Translation of the bone fragments may be impeded by non-completion of the osteotomy cut or inadequate release of soft tissue on lateral side of the first MT. Troughing refers to the situation where the cortical bone of one MT fragment wedges into the cancellous bone of the other fragment. Troughing is more common in elderly patients with osteoporotic bone. Troughing limits the amount of lateral translation of the capital fragment which leads to under-correction of HV. It also results in elevation and pronation of the first MT, causing dorsal malunion, transfer metatarsalgia, and HV recurrence (Fig. 7). ${ }^{36}$ Troughing occurs more commonly when the oblique limb of the osteotomy does not extend proximally and distally enough to include the metaphysis. ${ }^{36}$ The scarf osteotomy is not a pure diaphyseal osteotomy but begins at the distal metaphysis and ends in the proximal metaphysis. The metaphyseal portion of the cut allows for maximal cortical bone contact between the fragments during interfragmentary compression and fixation to prevent troughing. ${ }^{37}$

Avascular necrosis is uncommon in scarf osteotomies as long as the soft tissue attachment to the neck of the capital fragment, which contains the nutrient vessels to the MT head, is preserved. ${ }^{33}$ 

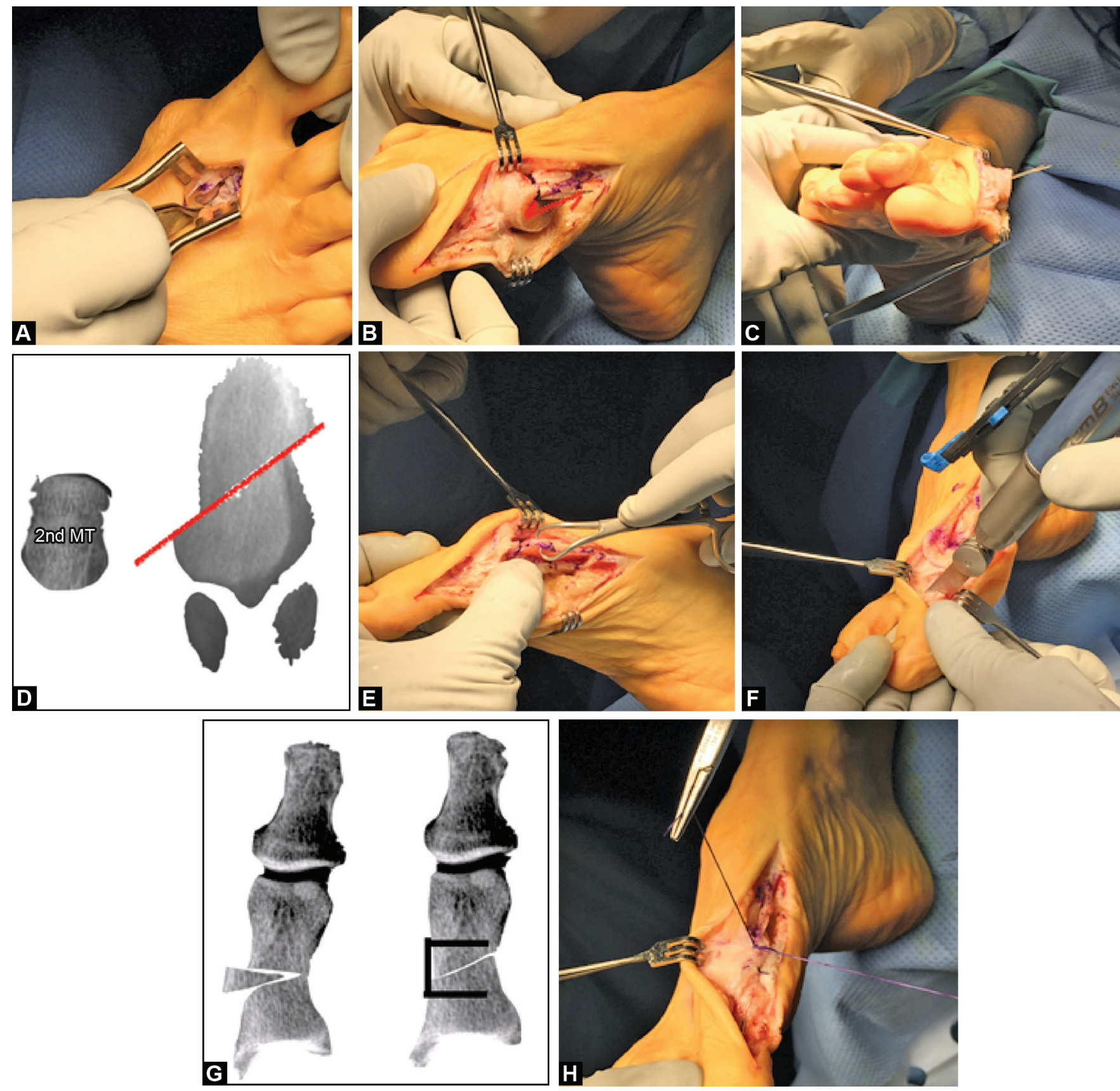

Figs $8 \mathrm{~A}$ to $\mathrm{H}$ : Intraoperative photographs and schematic diagrams illustrating authors' preferred approach to hallux valgus correction. The red lines on the photograph indicate the location and configuration of the first metatarsus (MT) osteotomy. (A) Lateral release of lateral sesamoidMT-proximal phalanx (PP) ligament complex through first web space incision; (B and C) Scarf osteotomy with saw blade in situ. Note the plantar angulation of the saw to depress the MT head to restore the weight-bearing function of the first metatarsus; (D) Schematic diagram illustrating the oblique plane of the scarf osteotomy; (E) Medial translation of the capital fragment with subsequent screw fixation (not shown); (F and G) Medial closing wedge osteotomy of first PP; $(\mathrm{H})$ Medial capsule plication

\section{Authors' Preferred Technique}

The authors recommend the following steps in the correction of $\mathrm{HV}$.

\section{Release of Lateral Sesamoid-Metatarsus-Proximal Phalanx Ligament Complex}

A lateral sesamoid-MT-PP ligament complex release is performed first. Through a lateral first web space incision, the lateral aspect of the first MTPJ is exposed. A longitudinal incision is made to release the lateral sesamoid-MT-PP ligament complex (Fig. 8A). Occasionally, the lateral head of flexor hallux brevis (FHB) is also divided in severe deformities. The objective is to release lateral pulling forces on the first PP exerted by the adductor hallucis, and to create a space for lateral translation of the head. There is a higher risk of AVN if an aggressive lateral soft tissue release is combined with a distal MT head/neck osteotomy. ${ }^{21}$ 


\section{Scarf First Metatarsus and Akin Proximal Phalanx Medial Closing Eedge Osteotomy}

After appropriate lateral soft tissue release, a scarf osteotomy is performed as described earlier. This is then fixed with two headless compression screws (Figs 8B to E). At this point, the foot is further assessed for residual deformity. There is often a component of hallux valgus interphalangeus ( $\mathrm{HVI})$ which can be addressed with a first PP medial closing wedge osteotomy first described by Akin (Figs $8 \mathrm{~F}$ and G). ${ }^{38}$ The Akin medial closing wedge osteotomy of the first PP corrects the $\mathrm{HVI}$ and also has the capacity to derotate the PP to correct for any pronation posture of the hallux (Fig. 8G). ${ }^{38,39}$ The osteotomy is typically fixed with a staple or a screw.

\section{Medial Capsule Plication}

Once the osteotomies and lateral release are completed the final correction is achieved through imbrication of the medial capsule (Fig. $8 \mathrm{H}$ ). At this juncture, the majority of the HV should have been corrected and the medial capsule repair is used mainly for finetuning of the final position of the hallux. Care should be taken not to overtighten the medial capsule which may lead to hallux varus. ${ }^{40}$

\section{Postoperative Recovery}

Patients are instructed to heel weight bear for 6 weeks. The leg is elevated for 2-3 weeks to reduce swelling and optimize incision healing. Toe spacers or bunion sleeves are used for 6 weeks.

\section{Patient Counseling}

Providing patients with a realistic expectation of surgery and recovery will help reduce patient misperception and dissatisfaction. Patients frequently underestimate the long recovery required for HV surgery. Patients are advised that recovering from HV surgery is akin to recovering from fractures, and may take many months. Prolonged swelling is expected. The foot has a tight soft tissue envelope and does not tolerate swelling well. In addition, gravity will constantly draw fluid to the feet. Patients are counseled that it will take a minimum of 2-3 months before swelling reduces to the point of being able to meaningfully wear dressy/fashionable shoes. For these reasons, rest and elevation for 2-3 weeks post-surgery until incisions heal is vitally important and must be emphasized.

\section{Complications}

Complications do occur with HV surgery even in the most experienced hands and the potential for this should be carefully discussed with patients prior to surgery. Recurrence of HV may occur when there is insufficient translation of the first MT osteotomy, failure to correct an increased DMAA or a pronation deformity, inadequate soft tissue releases or plication. ${ }^{41-44}$ Patients are counseled on the small risk of HV recurrence after surgery. These recurrences are often mild and the final result is frequently still an improvement from the preoperative alignment. Dorsal malunion is another potential complication that can be associated with proximal osteotomies with inadequate mechanical stability. ${ }^{45,46}$ The dorsally malunited first MT osteotomy will predispose to transfer metatarsalgia of the less rays. ${ }^{46}$ Hallux varus is uncommon but can occur if there has been an over-translation of the first MT, over-plication of the medial capsule, and aggressive lateral soft tissue release. ${ }^{40}$ The hallux is often slightly stiffer after surgery. However, this is rarely symptomatic.

\section{Salvage Procedures}

First MTPJ fusions may be used when HV occurs in conjunction with degenerative change, or as a salvage for the recurrent $\mathrm{HV}$ after initial surgery. ${ }^{27}$ First TMTJ fusions (Lapidus procedure) can also be considered in a revision situation to salvage recurrent $\mathrm{HV}^{47}$

\section{Minimally Invasive Surgery}

The rationale of MIS surgery is to use minimally invasive or percutaneous methods to correct HV. This minimizes soft tissue stripping, postsurgical swelling, and may expedite patient recovery. Minimally invasive surgery is growing in popularity and published results are favorable. ${ }^{3}$ It does require specialized equipment, such as low speed high torque burrs and a mini C-arm fluoroscopy (lower dose radiation), and these may not be readily available in some centers. The reader is directed to the paper on MIS surgery in this volume for further information.

\section{Conclusion}

Multiple methods and osteotomies are available to address HV and all can be effective in the right circumstances. Surgeons should choose techniques they are comfortable and confident with. Experience counts and there are nuances with any operation that can only learned through case volume and experience. For the less experienced surgeon, undertaking a proctorship with a colleague more experienced in the field will shorten the learning curve.

Regardless of which osteotomy the surgeon chooses, the principles of HV surgery remain the same. The aim being to reduce the IMA with an appropriate first MT osteotomy, correct any DMAA deformity with rotation of the first MT head, releasing contracted lateral sesamoid-MT-PP complex, a first PP medial closing wedge osteotomy to correct any residual $\mathrm{HVI}$, and a plication of the medial capsule of the first MTPJ. A clear understanding of these principles will guide our surgical decision-making.

Distal first MT osteotomies are appropriate for mild HV as they usually do not afford sufficient IMA correction. The scarf osteotomy coupled with first PP osteotomy can be used for both mild and severe deformity corrections. Distal metatarsal articular angle deformities can be addressed with rotation of the first MT head which can be achieved with the scarf osteotomy or biplanar chevron osteotomy. ${ }^{25,26}$ Osteotomies based on the proximal metaphysis can effect a large correction of the IMA, however, they can be more unstable. Soft tissue procedures, such as lateral release and medial plication, are needed in conjunction with osteotomies to achieve a desirable correction. A first PP osteotomy is used in the final stages of correction to address any residual HVI deformity.

Finally, the treatment of HV continues to evolve. Recently, there has been much emphasis on addressing the frontal rotational deformity (pronation and supination) of the first MT in HV. New techniques, such as proximal rotational metatarsal Osteotomy (PROMO), for HV have been devised to address the pronation deformity of the first ray. ${ }^{48}$ Experience with this is still in the early stages and further studies and time are required to establish the place of this procedure in the armamentarium of HV correction surgery.

\section{References}

1. Ray JJ, Friedmann AJ, Hanselman AE, et al. Hallux valgus. Foot and Ankle Orthopedics 2019;4(2):2473011419838500. 
2. Mann RA, Coughlin MJ. Hallux valgus-etiology, anatomy, treatment and surgical considerations. Clin Orthop Relat Res 1981;157(157): 31-41.

3. Holme TJ, Sivaloganathan SS, Patel B, et al. Third-generation minimally invasive chevron akin osteotomy for hallux valgus. Foot Ankle Int 2020;41(1):50-56. DOI: 10.1177/1071100719874360.

4. Gentili A, Masih S, Yao L, et al. Pictorial review: foot axes and angles. Br J Radiol 1996;69(826):968-974. DOI: 10.1259/0007-1285-69826-968.

5. Perera AM, Mason L, Stephens MM. The pathogenesis of hallux valgus. J Bone Joint Surg Am 2011;93(17):1650-1661. DOI: 10.2106/ JBJS.H.01630.

6. Dayton P, Kauwe M, Feilmeier M. Is our current paradigm for evaluation and management of the bunion deformity flawed? A discussion of procedure philosophy relative to anatomy. J Foot Ankle Surg 2015;54(1):102-111. DOI: 10.1053/j.jfas.2014.09.010.

7. Haines RW, MC DA. The anatomy of hallux valgus. J Bone Joint Surg $\mathrm{Br}$ 1954;36-B(2):272-293. DOI: 10.1302/0301-620X.36B2.272.

8. Alvarez R, Haddad RJ, Gould N, et al. The simple bunion: anatomy at the metatarsophalangeal joint of the great toe. Foot Ankle 1984;4(5):229-240. DOI: 10.1177/107110078400400502.

9. Coughlin MJ, Mann Award RA. Juvenile hallux valgus: etiology and treatment. Foot Ankle Int 1995;16(11):682-697. DOI: 10.1177/107110079501601104.

10. van Groningen B, van der Steen MC, Reijman M, et al. Outcomes in chevron osteotomy for hallux valgus in a large cohort. Foot (Edinb) 2016;29:18-24. DOI: 10.1016/j.foot.2016.09.002.

11. Austin DW, Leventen EO. A new osteotomy for hallux valgus: a horizontally directed "V" displacement osteotomy of the metatarsal head for hallux valgus and primus varus. Clin Orthop Relat Res 1981;157(157):25-30. DOI: 10.1097/00003086-198106000-00007.

12. Hawkins FB, Mitchell CL, Hedrick DW. Correction of hallux valgus by metatarsal osteotomy. J Bone Joint Surg Am 1945;27(3):387-394.

13. Wilson JN. Oblique displacement osteotomy for hallux valgus. J Bone Joint Surg Br 1963;45(3):552-556. DOI: 10.1302/0301-620X.45B3.552.

14. Mau C, Lauber HJ. Die operative behandlung des hallux valgus (Nachuntersuchungen). Deutsche Zeitschrift für Chirurgie 1926;197(1):361-377.

15. Saxena A, McCammon D. The ludloff osteotomy: a critical analysis. J Foot Ankle Surg 1997;36(2):100-105. DOI: 10.1016/s10672516(97)80053-1; discussion 59-60.

16. Borrelli AH. Modified scarf bunionectomy: Our experience in more than 1000 cases. J Foot Surg 1991;30:609.

17. Sammarco GJ, Brainard BJ, Sammarco VJ. Bunion correction using proximal chevron osteotomy. Foot Ankle 1993;14(1):8-14. DOI: 10.1177/107110079301400102.

18. Mann RA, Rudicel S, Graves SC. Repair of hallux valgus with a distal soft-tissue procedure and proximal metatarsal osteotomy. A longterm follow-up. J Bone Joint Surg Am 1992;74(1):124-129. DOI: 10.2106/00004623-199274010-00016.

19. SF A, MM B. Hallux Valgus in adolescents. J Bone Joint Surg Br 1960; 42-B(4):761-768.

20. Rothwell M, Pickard J. The chevron osteotomy and avascular necrosis. Foot (Edinb) 2013;23(1):34-38. DOI: 10.1016/j.foot.2012.10.001.

21. Rath B, Notermans HP, Franzen J, et al. The microvascular anatomy of the metatarsal bones: a plastination study. Surg Radiol Anat 2009;31(4):271-277. DOI: 10.1007/s00276-008-0441-3.

22. Easley ME, Kiebzak GM, Davis WH, et al. Prospective, randomized comparison of proximal crescentic and proximal chevron osteotomies for correction of hallux valgus deformity. Foot Ankle Int 1996;17(6):307-316. DOI: 10.1177/107110079601700603.

23. Coughlin MJ. Hallux valgus in men: effect of the distal metatarsal articular angle on hallux valgus correction. Foot Ankle Int 1997;18(8):463-470. DOI: 10.1177/107110079701800802.

24. Lee KT, Park YU, Jegal $H$, et al. Deceptions in hallux valgus: what to look for to limit failures. Foot Ankle Clin 2014;19(3):361-370. DOI: 10.1016/j.fcl.2014.06.003.

25. Nery C, Barroco R, Ressio C. Biplanar chevron osteotomy. Foot Ankle Int 2002;23(9):792-798. DOI: 10.1177/107110070202300903.
26. Murawski CD, Egan CJ, Kennedy JG. A rotational scarf osteotomy decreases troughing when treating hallux valgus. Clin Orthop Relat Res 2011;469(3):847-853. DOI: 10.1007/s11999-010-1647-3.

27. Grimes JS, Coughlin MJ. First metatarsophalangeal joint arthrodesis as a treatment for failed hallux valgus surgery. Foot Ankle Int 2006;27(11):887-893. DOI: 10.1177/107110070602701104.

28. Feilmeier M, Dayton P, Wienke JC,Jr. Reduction of intermetatarsal angle after first metatarsophalangeal joint arthrodesis in patients with hallux valgus. J Foot Ankle Surg 2014;53(1):29-31. DOI: 10.1053/j. jfas.2013.09.012.

29. Chraim M, Bock P, Alrabai HM, et al. Long-term outcome of first metatarsophalangeal joint fusion in the treatment of severe hallux rigidus. Int Orthop 2016;40(11):2401-2408. DOI: 10.1007/s00264-0163277-1.

30. Schmid T, Krause F. The modified lapidus fusion. Foot Ankle Clin 2014;19(2):223-233. DOI: 10.1016/j.fcl.2014.02.005.

31. Mallette JP, Glenn CL, Glod DJ. The incidence of nonunion after lapidus arthrodesis using staple fixation. J Foot Ankle Surg 2014;53(3):303-306. DOI: 10.1053/j.jfas.2013.09.004.

32. Patel S, Ford LA, Etcheverry J, et al. Modified lapidus arthrodesis: rate of nonunion in 227 cases. J Foot Ankle Surg 2004;43(1):37-42. DOI: 10.1053/j.jfas.2003.11.009.

33. Barouk LS. Scarf osteotomy for hallux valgus correction. Local anatomy, surgical technique, and combination with other forefoot procedures. Foot Ankle Clin 2000;5(3):525-558.

34. Barouk LS. Forefoot Reconstruction 2006. p. 388.

35. Coetzee JC, Rippstein P. Surgical strategies: scarf osteotomy for hallux valgus. Foot Ankle Int 2007;28(4):529-535. DOI: 10.3113/FAl.2007.0529.

36. Coetzee JC. Scarf osteotomy for hallux valgus repair: the dark side. Foot Ankle Int 2003;24(1):29-33. DOI: 10.1177/107110070302400104.

37. Lee $\mathrm{SC}$, Hwang $\mathrm{SH}, \mathrm{Nam} \mathrm{CH}$, et al. Technique for preventing troughing in scarf osteotomy. J Foot Ankle Surg 2017;56(4):822-823. DOI: 10.1053/j.jfas.2017.01.051.

38. Akin OF. The treatment of hallux valgus: a new operative procedure and its results. Med Sentinel 1925;33:678-679.

39. Douthett SM, Plaskey NK, Fallat LM, et al. Retrospective analysis of the akin osteotomy. J Foot Ankle Surg 2018;57(1):38-43. DOI: 10.1053/j. jfas.2017.06.026.

40. Donley BG. Acquired hallux varus. Foot Ankle Int 1997;18(9):586-592. DOI: $10.1177 / 107110079701800911$.

41. Bock P, Kluger R, Kristen KH, et al. The scarf osteotomy with minimally invasive lateral release for treatment of hallux valgus deformity: intermediate and long-term results. J Bone Joint Surg Am 2015;97(15):1238-1245. DOI: 10.2106/JBJS.N.00971.

42. Jeuken RM, Schotanus MG, Kort NP, et al. Long-term follow-up of a randomized controlled trial comparing scarf to chevron osteotomy in hallux valgus correction. Foot Ankle Int 2016;37(7):687-695. DOI: $10.1177 / 1071100716639574$

43. Kilmartin $\mathrm{TE}, \mathrm{O}^{\prime}$ Kane $\mathrm{C}$. Combined rotation scarf and akin osteotomies for hallux valgus: a patient focussed 9 year follow up of 50 patients. J Foot Ankle Res 2010;3(1):2. DOI: 10.1186/1757-1146-3-2.

44. Pentikainen I, Ojala R, Ohtonen $\mathrm{P}$, et al. Preoperative radiological factors correlated to long-term recurrence of hallux valgus following distal chevron osteotomy. Foot Ankle Int 2014;35(12):1262-1267. DOI: $10.1177 / 1071100714548703$.

45. Merkel KD, Katoh Y, Johnson Jr EW, et al. Mitchell osteotomy for hallux valgus: long-term follow-up and gait analysis. Foot Ankle 1983;3(4):189-196. DOI: 10.1177/107110078300300402.

46. Jones $C$, Coughlin M, Villadot $R$, et al. Proximal crescentic metatarsal osteotomy: the effect of saw blade orientation on first ray elevation. Foot Ankle Int 2005;26(2):152-157. DOI: 10.1177/107110070502 600207.

47. Coetzee JC, Resig SG, Kuskowski M, et al. The Lapidus procedure as salvage after failed surgical treatment of hallux valgus: a prospective cohort study. J Bone Joint Surg Am 2003;85(1):60-65. DOI: 10.2106/00004623-200301000-00010.

48. Wagner P, Wagner E. Proximal rotational metatarsal osteotomy for hallux valgus (PROMO):short-term prospective case series with a novel technique and topic review. 2018;3(3):2473011418790071. 